\title{
Étude biologique et biochimique du déterminisme de la croissance rythmique du chêne pédonculé (Quercus robur $\mathrm{L}$ ). Effets de l'ablation des feuilles
}

\author{
P Barnola 1*, D Alatou 2, A Lacointe ${ }^{3}, \mathrm{~S}$ Lavarenne 4 \\ 1 Université de Nancy l, laboratoire de biologie des ligneux, BP 239, \\ 54506 Vandceuvre-lès-Nancy Cedex, France; \\ 2 Université de Constantine, Institut des sciences de la nature, \\ route de Ain-El Bey, Constantine, Algérie; \\ 3 INRA, bioclimatologie, Domaine de Crouelle 63039 Clermont-Ferrand; \\ 4 CNRS UA 45, Université de Clermont II, laboratoire de phytomorphogenèse \\ 4, rue Ledru, 63038 Clermont-Ferrand Cedex, France
}

(Reçu le 10 janvier 1990; accepté le 22 août 1990)

\begin{abstract}
Résumé - La croissance du chêne pédonculé cultivé à $25^{\circ} \mathrm{C}\left( \pm 1^{\circ} \mathrm{C}\right)$ en jour long, est parfaitement rythmique. La suppression des limbes dont la taille est inférieure à $10 \mathrm{~mm}$, la transforme en croissance continue. La suppression des feuilles d'un étage $n$ ayant atteint leur taille définitive, provoque une très forte réduction en longueur de l'étage $n+1$ lorsque celui-ci se développe. Au cours d'un flush, l'incorporation de la ${ }^{14} \mathrm{C}$-DMO (5-5' diméthyloxazolidine 2-4 dione), acide faible lipophile, par le bourgeon terminal, les tissus de l'axe sous-jacent au bourgeon terminal et les feuilles en croissance varie au cours du temps chez une plante intacte. Les résultats obtenus avec la fourniture de ${ }^{14} \mathrm{CO}_{2}$ au cours de la troisième vague de croissance confirment les résultats obtenus avec la ${ }^{14} \mathrm{C}-\mathrm{DMO}$. Dans le cas d'une plante dont la croissance est devenue continue à la suite de l'ablation des très jeunes feuilles, le bourgeon accumule toujours plus de ${ }^{14} \mathrm{C}$-DMO que les entre-nceuds. Dans le cas de plantes où seule la croissance en longueur de l'étage est réduite par ablation des feuilles ayant atteint leur taille définitive, l'absorption de la ${ }^{14} \mathrm{C}$-DMO est identique à celle des témoins. L'ensemble des résultats permet de proposer une hypothèse explicative de la croissance rythmique du chêne pédonculé, basée sur la notion de puits.
\end{abstract}

ablation des feuilles / ${ }^{14} \mathrm{C}$-DMO / ${ }^{14} \mathrm{CO}_{2} /$ croissance rythmique / Quercus robur

Summary - A biological and biochemical study of the factors determining rhythmical growth in pedunculate oak (Quercus robur). The effects of leaf removal. Quercus robur $L$ showed perfect rhythmical growth when it was cultivated under controlled conditions at $25^{\circ} \mathrm{C}\left( \pm 1^{\circ} \mathrm{C}\right)$, with long days or continuous light at $80 \mu \mathrm{mol} \cdot \mathrm{m}^{-2} \cdot \mathrm{s}^{-1}$. It was characterized by a regular succession of flushes. One flush lasted for 3 weeks: the first 2 weeks were the growing period and the last was the rest period. It was a false rest period because the activity of the apical meristem did not stop. A flush was characterized by the succession of scales and leaves, and by a succession of long and short internodes. The rhythmical growth is inhibited when $10 \mathrm{~mm}$ leaves are removed. Then continuous growth takes place. The apical meristem keeps producing a primortium. If a primortium is not removed it always gives a leaf and never a scale. The removal of the leaves of a flush at their adult size produces a strong reduction in the length of the next flush. During a flush, the intracellular concentration of the ${ }^{14} \mathrm{C}$-DMO (5 dimethyl oxazolidine 2-4 dione), a weak and lipophylacid, in the terminal bud and in its

\footnotetext{
* Correspondance et tirés à part
} 
adjacent axial tissues, and in leaves, varies in an untouched plant. The same variations are repeated for every flush. The results with a supply of ${ }^{14} \mathrm{CO}_{2}$ confirm the results with the ${ }^{14} \mathrm{C}$-DMO.

In the case of plants with continuous growth caused by the regular removal of young leaves, the intracellular concentration of ${ }^{14} \mathrm{C}$-DMO always remains higher in the terminal bud than in the internodes. There is no change in plants where only the adult leaves of a flush are removed.

Our results allow us to put forward a nutritional hypothesis of the rhythmical growth of Quercus robur $L$. The rhythmical growth is the result of the relationships between 3 elements which are: the apical meristem, the axial tissues bearing the terminal bud, and the very young leaves. If one element, the axial tissues or the young leaves becomes stronger than the others, the growth is changed. Then it is possible that the internodes become short and the primordium produces scales.

leaf removal $/{ }^{14} \mathrm{C}-\mathrm{DMO} /{ }^{14} \mathrm{CO}_{2} /$ rhythmical growth / Quercus robur

\section{INTRODUCTION}

Les chênes, qu'ils soient en régions tempérées ou méditerranéennes, sont connus pour leurs vagues successives de croissance, ou pousses de la Saint-Jean, qu'ils peuvent réaliser durant leur période annuelle de végétation (Lavarenne, 1965). II est possible de reproduire parfaitement cette croissance en chambre climatisée et température constante $\left(28^{\circ} \mathrm{C}\right)$. Dans ces conditions, jusqu'à 16 vagues ou flushs sont obtenus en un an, alors qu'on en observe au maximum 3 dans la nature chez le chêne pédonculé.

Les feuilles ont un rôle dans cette croissance rythmique. En effet, l'ablation des feuilles de moins de $10 \mathrm{~mm}$ instaure une croissance continue avec des entrenœeuds réduits et des feuilles à limbe entier pour celles dont le développement est permis (Payan, 1982; Champagnat et al, 1986).

Compte tenu des résultats acquis chez le chêne pédonculé, nous avons voulu analyser plus précisément l'influence des feuilles jeunes et des feuilles ayant atteint leur taille définitive, sur la croissance des jeunes plants cultivés en conditions de croissance non limitantes. Le but de l'étude s'inscrit dans le cadre d'un objectif fondamental : connaître les mécanismes de la croissance rythmique du chêne pédonculé et déterminer si ces mécanismes sont à l'origine de la croissance rythmique qui est un aspect général de la croissance des arbres.

Aux techniques biologiques classiques d'ablation des feuilles, on a adjoint les techniques biochimiques suivantes :

- l'étude de l'incorporation intracellulaire de la 5,5'-diméthyloxazolidine-2,4 dione ou ${ }^{14} \mathrm{C}$-DMO. La ${ }^{14} \mathrm{C}$-DMO a été employée pour évaluer le $\mathrm{pH}$ intracellulaire de cellules végétales cultivées isolément (Kurkdjian et al, 1978). Elle possède les caractéristiques d'absorption, par les cellules végétales, de molécules comme l'ABA (Mokhbi, 1988) et l'AlA. Sa plus grande accumulation dans des organes ou des territoires cellulaires va de pair avec une capacité d'absorption plus forte d'éléments influençant la croissance, ce qui permettrait d'assimiler ces territoires à des «puits» (Gendraud et Lafleuriel, 1983; Auriac et Tort, 1985; Auriac, 1987; Pezet-Si Mohamed, 1987).

- le marquage au ${ }^{14} \mathrm{C}-\mathrm{CO}_{2}$, technique qui fut employée aussi par Dickson (1989) pour suivre les caractéristiques de la croissance par vagues du chêne rouge.

\section{MATÉRIEL ET MÉTHODES}

\section{Matériel végétal}

Les glands de chêne pédonculé (Quercus robur L : Quercus pédonculata Ehrh) récoltés au mois 
d'octobre au-dessous d'un même semencier situé à Lezoux (Puy-de-Dôme), sont stratifiés et mis en conservation à $1^{\circ} \mathrm{C}$. Les semences sont débarrassées de leurs enveloppes séminales et placées $24 \mathrm{~h}$ sous l'eau courante. La germination est réalisée en 15 jours à $16 \pm 1^{\circ} \mathrm{C}$ dans de la vermiculite humide. Les germinations, dont la radicule mesure de 8 à $10 \mathrm{~cm}$, sont alors transférées sur un dispositif de culture hydroponique en chambre climatisée à $25 \pm 1^{\circ} \mathrm{C}$ en jour long de $16 \mathrm{~h}$. L'intensité de l'éclairement, émanant d'un plafond lumineux constitué de tubes fluorescents (15 tubes lumière du jour TF65-58 W, 10 tubes lumière du jour de lune TFRS 65/LjL, 5 tubes fluorescents GRO-LUX TF65 W et de 12 lampes à incandescence $15 \mathrm{~W}$ ), est de 80 $\mu \mathrm{mol} \cdot \mathrm{m}^{-2} \cdot \mathrm{s}^{-1}$ à la base des plantes.

Dans ces conditions, l'axe orthotrope du chêne pédonculé est constitué d'une succession ininterrompue d'unités sructurales identiques qui se mettent en place au cours de vagues de croissance ou "flushs". Les vagues de croissance durent en moyenne 2 semaines, séparées par une période de repos d'environ une semaine. A la fin de la période de repos, le bourgeon apical gonfle et engendre un axe avec à sa base des ensembles foliaires écailleux. Les limbes assimilateurs se développent ensuite pour donner les feuilles lobées du chêne (fig 1). La vague de croissance se termine par la mise en place de 3 ensembles foliaires au limbe de plus en plus atrophié ( $1 \mathrm{à} 4 \mathrm{~mm}$ ) et aux stipules de plus en plus courtes et larges; ce sont les limbes avortés qui recouvrent le bourgeon terminal. En résumé, une unité structurale est composée de bas en haut (fig 1) :

- d'ensembles foliaires réduits aux stipules; les derniers formés sont situés au niveau d'entrenceuds longs;

- d'ensembles foliaires aux stipules rapidement caduques avec limbe assimilateur, les deux premiers sont associés à des entre-nœuds longs; les suivants sont groupés à la partie supérieure de l'étage du fait de la réduction de l'allongement des entre-nœuds;

- d'ensembles foliaires aux stipules devenant écailleuses avec un petit limbe bien net mais qui se dessèche rapidement: ce sont les feuilles à limbe avorté. Elles terminent un étage et seront à la base de l'étage suivant.

\section{Techniques biologiques}

Toutes les expériences réalisées ont pour but de modifier la mise en place du troisième étage.

\section{Ablation des jeunes feuilles}

\section{Totale}

La suppresion de ces feuilles commence à partir du $6^{\theta}$ jour du flush lorsque le bourgeon apical

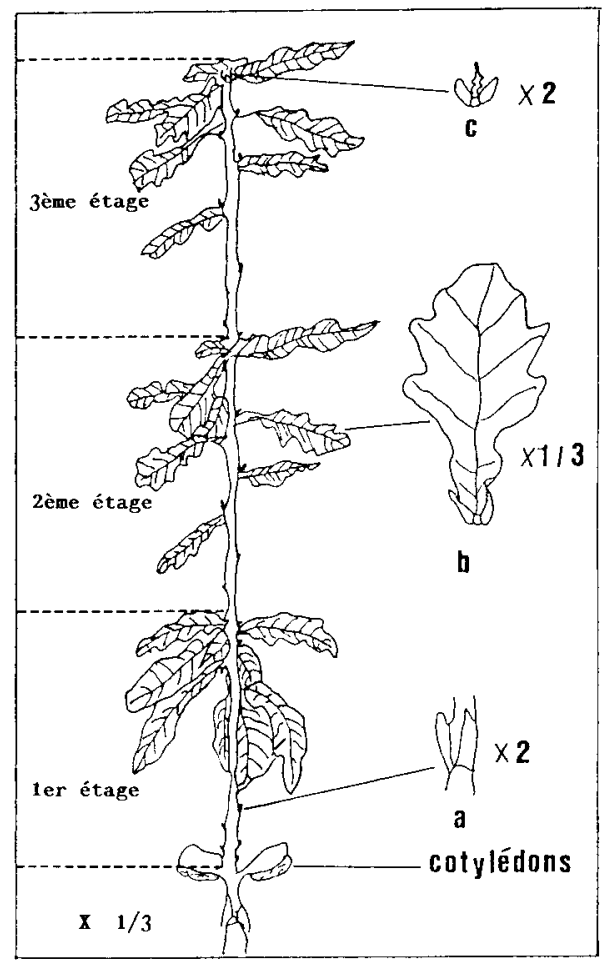

Fig 1. Schéma d'un plant de chêne pédonculé issu de germination, âgé de 2 mois, au stade de la $3^{\theta}$ vague de croissance, cultivé à $25^{\circ} \mathrm{C}$ en jours longs de $16 \mathrm{~h}$. Les différents ensembles foliaires sont représentés : a, stipules écailleuses; b, feuille stipulée à limbe assimilateur; c, feuille stipulée à limbe avorté. 
est épanoui et laisse voir un ensemble de limbes assimilateurs de petite taille. Le méristème apical émet alors continuellement et régulièrement des ébauches foliaires qui sont supprimées tous les 2 jours. L'expérience dure 32 jours au cours desquels ne s'installe aucune période de repos.

\section{Partielle}

L'ablation porte tout d'abord sur les jeunes feuilles de taille inférieure à $10 \mathrm{~mm}$, au $6^{\Theta}$ jour de la $3^{\theta}$ vague de croissance, puis $1,2,3$ ou 4 feuilles sont conservées. Les autres jeunes feuilles, qui sont initiées ensuite, sont supprimées régulièrement tous les 2 jours. L'expérience dure 4 semaines.

\section{Ablation des feuilles adultes}

\section{Totale}

Tous les limbes assimilateurs adultes à la fin du repos apparent du $2^{\ominus}$ étage sont supprimés $\left(20^{\circ}\right.$ jour du flush). La plante poursuit son développement et l'observation est faite durant la $3^{\theta}$ et $4^{\theta}$ vague de croissance.

\section{Partielle}

L'expérience consiste à enlever $50 \%$ des limbes assimilateurs adultes, à la fin de la période du repos apparent $\left(20^{\ominus}\right.$ jour du $2^{\mathrm{e}}$ flush). Deux cas sont examinés selon que la suppression de la moitié des feuilles est réalisée dans la partie proximale ou distale de l'étage. Le développement de la plante est suivi jusqu'à la $4^{\mathrm{e}}$ vague de croissance.

\section{Techniques biochimiques}

\section{Pénétration et accumulation de la ${ }^{14}$ C-DMO}

La technique consiste à évaluer la pénétration dans les cellules d'un acide faible lipophile, la 5,5-diméthyloxazolidine-2,4 dione $\left[2-{ }^{-14} \mathrm{C}\right)$ ou ${ }^{14} \mathrm{C}$-DMO qui traverse la membrane uniquement sous sa forme non dissociée. La sonde se dissocie à l'intérieur et à l'extérieur de ia cellule selon le $\mathrm{pH}$ de chaque compartiment jusqu'à l'obtention d'un équilibre de diffusion. Cette technique, mise au point sur des cultures de cellules d'érable isolées (Kurkdjian et Guern, 1978) fut adaptée ensuite aux parenchymes de topinambour (Gendraud et Lafleuriel, 1983), de crosne du Japon (Auriac, 1987) et aux tissus de végétaux ligneux (le chêne pédonculé : Barnola et al, 1986; Alatou et al, 1989; le châtaignier : PezetSi-Mohamed, 1987).

Les territoires et organes suivants ont été étudiés :

- le bourgeon apical débarassé de ses écailles;

- la section d'axe sous-jacente au bourgeon terminal. Elle est constituée essentiellement par $90 \%$ de parenchymes médullaire et cortical;

- un fragment de limbe assimilateur.

Ces fragments sont placés séparément en incubation dans un milieu de KNOPP dilué au quart, renfermant $3,7 \mathrm{kBq}$ de ${ }^{14} \mathrm{C}$-DMO et 27,75 $\mathrm{kBq}$ de ${ }^{3} \mathrm{H}$-insuline. L'incubation a lieu à $25^{\circ} \mathrm{C}$ à l'obscurité pendant $16 \mathrm{~h}$. Elle est d'une durée suffisante pour atteindre l'équilibre de diffusion de la DMO. La solution radioactive absorbée est extraite par la méthode de Jefford et Edelman (1961) puis mesurée par scintillation liquide. La concentration intracellulaire (Ci) représente le rapport Qi/Vi de la quantité intercellulaire (Qi) de ${ }^{14} \mathrm{C}$-DMO au volume intracellulaire (Vi). Ce dernier est calculé directement par la différence entre le volume total de l'échantillon et le volume intercellulaire. Celui-ci est assimilé au volume accessible à l'insuline ${ }^{3} \mathrm{H}$. Qi est la quantité totale de ${ }^{14} \mathrm{C}$-DMO absorbée par l'échantillon, diminuée de la quantité de ${ }^{14} \mathrm{C}$-DMO métabolisée. Cette dernière est déterminée après chromatographie sur plaque de cellulose d'une aliquote de l'extrait dans le solvant isopropanol/ ammoniaque/eau $(8 / 1 / 1, \mathrm{v} / \mathrm{v} / \mathrm{v})$. La concentration extracellulaire ( $\mathrm{Ce}$ ) est déterminée avec une aliquote du milieu d'incubation.

Le rapport $\mathrm{Ci} / \mathrm{Ce}$ en ${ }^{14} \mathrm{C}$-DMO est calculé pour chaque échantillon et rend compte pour chacun d'entre eux et à chaque étape considérée, de la facilité de pénétration de la DMO dans les cellules. Plus le rapport est élevé, plus la DMO s'accumule dans les cellules, élevant le $\mathrm{pH}$ intracellulaire (Candelier, 1989).

Cette technique biochimique a été appliquée pour l'ablation systématique des jeunes feuilles dont la taille est inférieure à $10 \mathrm{~mm}$ et des feuilles du deuxième étage ayant atteint leur longueur définitive. 


\section{Marquage au ${ }^{14} \mathrm{CO}_{2}$ de jeunes plants de chêne pédonculé au cours de la $3^{e}$ vague de croissance}

Le marquage au ${ }^{14} \mathrm{CO}_{2}$ est une technique qui a été utilisée sur de nombreux végétaux ligneux notamment : pommier (Hansen et Grauslund, 1973; Kandiah, 1979), peuplier (Dickson et Nelson, 1982), noyer (Lacointe, 1989), épicéa commun (Langenfeld-Heyser, 1987), chêne rouge (Dickson, 1989). Le principe consiste à faire libérer du ${ }^{14} \mathrm{CO}_{2}$ par simple réaction chimique entre un acide et un bicarbonate de sodium marqué au ${ }^{14} \mathrm{C}$. Pour une activité totale de $500 \mu \mathrm{Ci}$, $25 \mathrm{ml} \mathrm{d} \mathrm{H}_{2} \mathrm{SO}_{4}$ aे $10 \%$ et $0,5 \mathrm{ml}$ d'une solution basique de ${ }^{14} \mathrm{CO}_{3} \mathrm{Na}_{2}$ à $1 \mathrm{mCi} / \mathrm{ml}$ (CEA, CMM53B) sont utilisés pour assurer le dégagement du gaz radioactif. Celui-ci est aspiré par une pompe et distribué dans la chambre d'assimilation. A la fin du marquage, le ${ }^{14} \mathrm{CO}_{2}$ encore présent est piégé dans une solution de $\mathrm{KOH}$. Le circuit est purgé én $30 \mathrm{~min}$.

\section{Conditions climatiques de la chambre d'as- similation}

La température de l'atmosphère varie entre 20 et $24^{\circ} \mathrm{C}$. La densité de flux de photons mesurée par quantum-sensor Delta T, 400 à $700 \mathrm{~nm}$ est de $260 \mu \mathrm{mol} \cdot \mathrm{m}^{-2} \cdot \mathrm{s}^{-1}$ à $40 \mathrm{~cm}$ au-dessus du sol. Elle émane d'un plafond lumineux constitué de 30 tubes Osram Fluora L 58 W/77.

\section{Protocole expérimental}

Les plants ${ }^{14} \mathrm{C}$ de chêne pédonculé cultivés en conditions contrôlées à $25^{\circ} \mathrm{C}$ sont transférés en chambre d'assimilation aux étapes $1,8,14$ et 18 jours de la $3^{\theta}$ vague de croissance, durant $5 \mathrm{~h}$.

Le matériel végétal prélevé après le marquage correspond au bourgeon apical débarrassé de ses écailles, à une portion d'environ 5 à 8 $\mathrm{mm}^{3}$ de l'axe sous-jacent à celui-ci, à des fragments de limbes assimilateurs de l'étage en formation et de celui qui le précède. Les échantillons sont placés séparément dans l'hydroxyde d'hyamine, qui permet d'extraire la radioactivité après $16 \mathrm{~h}$ d'incubation par simple digestion du matériel végétal. La radioactivité est mesurée par scintillation liquide.

Une aliquote de chaque extrait est déposée dans une fiole contenant $5 \mathrm{ml}$ de scintillant ou liquide de Bray.

\section{Echantillonnage et expression des ré- sultats}

\section{Ablations des feuilles et mesures d'in- corporation de la DMO}

Les traitements ont été faits sur des séries de 6 ou 12 plants présentant les mêmes stades de développement. Les expériences ont été répétées 3 fois avec, pour chaque répétition, les mêmes résultats. En ce qui concerne les résultats morphologiques, ils sont fournis sous forme . d'histogrammes individuels représentant les longueurs des entre-nœuds et des limbes. Pour l'incorporation de la DMO, nous donnons la moyenne, accompagnée de l'erreur standard, des résultats obtenus avec une série de plants traités.

\section{Marquage au ${ }^{14} \mathrm{CO}_{2}$}

Pour chacun des 14 individus traités à chaque étape, les valeurs du radiocarbone (dpm $\mathrm{mg}^{-1}$ de matière fraîche (MF) de l'extrait) rendent compte pour chaque échantillon, de la quantité d'assimilats accumulée produite par photosynthèse des feuilles de chênes soumises à un flux de ${ }^{14} \mathrm{CO}_{2}$.

\section{RÉSULTATS}

\section{Ablation régulière des jeunes feullles de moins de $10 \mathrm{~mm}$ de longueur et in- corporation de la ${ }^{14} \mathrm{C}$-DMO}

La croissance des plantes est ininterrompue, les primordia sont engendrés régulièrement. Si l'un d'entre eux est conservé, il donne toujours une feuille à limbe assimilateur. Les entre-nœuds restent très courts, l'allongement moyen est de 1 à 2 $\mathrm{mm}$ par jour (fig 2).

Chez toutes les plantes dont les jeunes feuilles sont supprimées, à 10, 22, 28 et 32 jours de croissance, le bourgeon terminal accumule davantage la ${ }^{14} \mathrm{C}$-DMO que les 


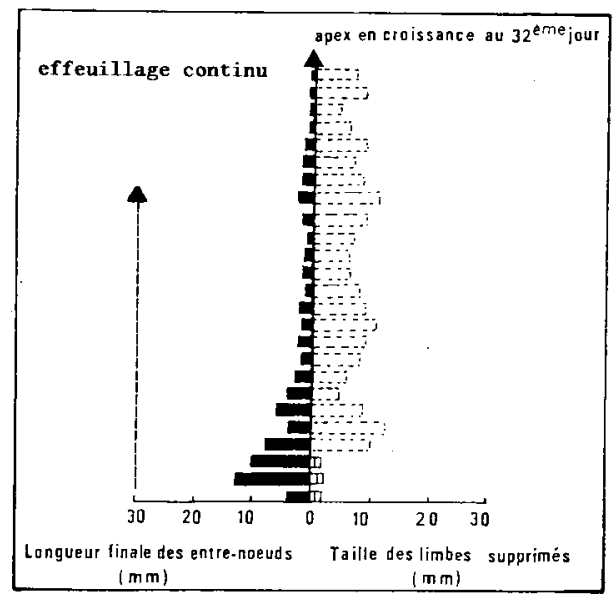

Fig 2. Ablation continue des jeunes limbes dont la taille est inférieure à $10 \mathrm{~mm}$ au cours de la $3^{\circ}$ vague de croissance chez le chêne pédonculé cultivé à $25^{\circ} \mathrm{C}$ en jours longs de $16 \mathrm{~h}$. La croissance est suivie pendant 32 jours. L'apex a produit 22 limbes assimilateurs (tirets) associés à des entre-nœuds courts (traits pleins), insérés sur l'axe caulinaire du bas vers le haut de

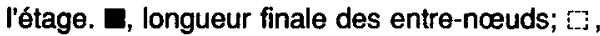
taille des limbes au moment de leur ablation; $\mathbb{\sigma}$, écailles; $\bullet$, bourgeon apical.

tissus de l'axe immédiatement sousjacents (fig 3). Ce résultat est très différent de celui observé chez les plantes dont seules les feuilles ayant atteint leur taille définitive sont supprimées.

Si une ou deux feuilles sont conservées, la croissance du chêne reste continue. La surface foliaire est multipliée par 2 et 1,5 respectivement. L'entre-nœud sousjacent au limbe assimilateur conservé s'allonge. A partir de 3 ou 4 feuilles maintenues, le plastochrone apparent diminue et atteint la valeur de 0,3 feuille par jour. Le bourgeon apical marque alors un arrêt, la croissance rythmique est rétablie. L'étage qui suit est comparable à celui du témoin intact.

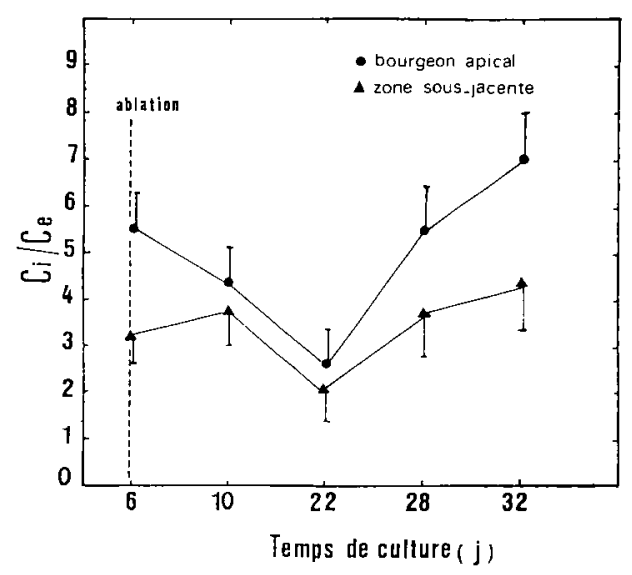

Fig 3. Evolution du rapport $\mathrm{Ci} / \mathrm{Ce}$ de la concentration intracellulaire à la concentration extracellulaire en DMO en fonction du temps en jours au cours de la croissance continue, obtenue après ablation des jeunes feuilles chez le chêne pédonculé cultivé à $25^{\circ} \mathrm{C}$ en jours longs. bourgeon apical. $\boldsymbol{\Delta}$, axe sous-jacent au bourgeon apical. Ne sont figurées que les moitiés des écarts à la moyenne pour plus de lisibilité des graphiques. Chaque valeur représente la moyenne de 6 mesures \pm erreur standard.

\section{Ablation des feuilles du $2^{\circ}$ étage; obser- vation du développement du $3^{\circ}$ étage et incorporation de la ${ }^{14} \mathrm{C}$-DMO}

L'ablation totale de toutes les feuilles ayant atteint leur surface définitive à la fin du repos apparent du $2^{e}$ étage, conduit à la formation d'un $3^{\theta}$ étage présentant une réduction notable de sa longueur. II s'agit d'un "mini-étage", car les entre-nœuds les plus longs chez les témoins sont aussi les plus longs dans le "mini-étage" (fig 4). La composition spatiale en ensembles foliaires du «mini-étage» reste la même que chez les témoins et la surface foliaire est peu affectée. Une diminution de deux jours de la phase d'allongement est observée. 


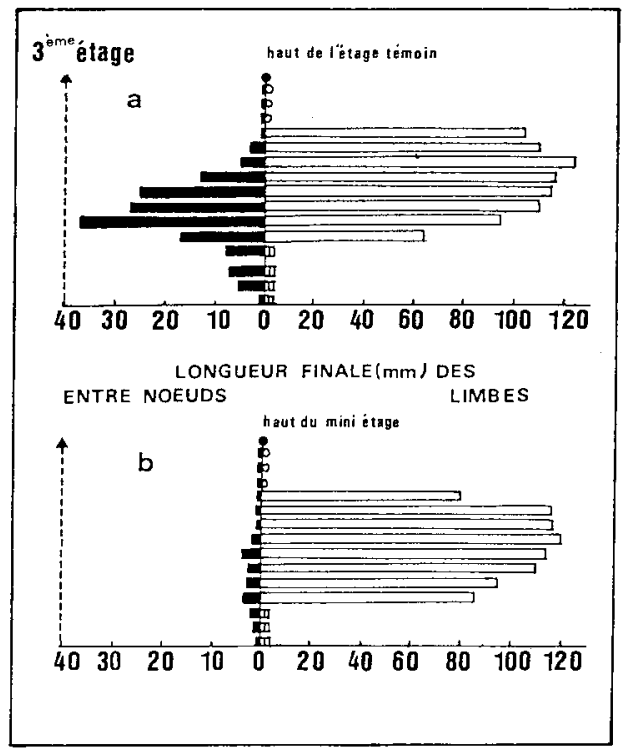

Fig 4. Influence de l'ablation totale des limbes adultes du $2^{\circ}$ étage sur le développement du $3^{\theta}$ étage chez le chêne pédonculé cultivé à $25^{\circ} \mathrm{C}$ en jours longs de $16 \mathrm{~h}$. $a$, plant intact; $b$, plant ayant subi l'ablation des feuilles. Sont représentés les entre-nœuds associés aux différents ensembles foliaires suivant leur insertion sur l'axe caulinaire du bas vers le haut de l'étage. $\square$, longueur finale des entre-nœuds; $\square$, longueur finale des limbes assimilateurs; $\square$, écailles; $O$, limbes avortés; $\bullet$, bourgeon apical.

La vague de croissance qui suit le «miniétage" est identique à celle que l'on observe chez le témoin non effeuillé.

Lorsque la moitié des feuilles du $2^{\mathrm{e}}$ étage est conservée, la $3^{e}$ vague de croissance est de longueur normale. Que l'ablation concerne la partie inférieure de l'étage $n$, ou sa partie supérieure, les feuilles restantes, soit $50 \%$ de l'effectif initial suffisent à assurer une élongation de l'étage $n+1$ comparable au témoin. La réduction en longueur de l'étage se manifeste quand
$75 \%$ de l'effectif initial des feuilles est supprimé.

Malgré les modifications morphologiques observées (obtention d'un «miniétage"), l'ablation totale des feuilles du $2^{\theta}$ étage ne conduit pas à une modification de la concentration intracellulaire en DMO par rapport aux témoins (fig 5). Le bourgeon apical présente un rapport $\mathrm{Ci} / \mathrm{Ce}$ qui s'élève rapidement depuis le début de la vague de croissance jusqu'au $8^{\theta}$ jour. II est supérieur à celui des tissus de l'axe sousjacent pendant la $1^{\text {re }}$ semaine de croissance, puis il décroît fortement entre le $8^{e}$ et le $11^{\theta}$ jour (arrêt de l'allongement caulinaire), et se stabilise jusqu'à la fin de la vague ( $21^{\mathrm{e}}$ jour). Le rapport $\mathrm{Ci} / \mathrm{Ce}$ dans les tissus de l'axe sous-jacent, d'abord inférieur à celui du bourgeon apical, augmente aussi jusqu'au $11^{\ominus}$ jour; à partir de là, le rapport devient plus grand pour les tissus de l'axe que pour ceux du bourgeon apical. Les limbes assimilateurs restés présents, montrent une augmentation accentuée du rapport $\mathrm{Ci} / \mathrm{Ce}$ du $8^{\mathrm{e}}$ au $11^{\mathrm{e}}$ jour, situation identique à celle obtenue chez les plantes témoins.

L'absence de modifications biochimiques par la technique de la ${ }^{14} \mathrm{C}-\mathrm{DMO}$, malgré la présence d'un “mini-étage», sera discutée.

Répartition des assimilats chez de jeunes chênes pédonculés soumis à un pulse de ${ }^{14} \mathrm{CO}_{2}$

La distribution du carbone fixé par photosynthèse sous forme d'assimilats est suivie au cours de la $3^{e}$ vague de croissance du chêne pédonculé cultivé à $25^{\circ} \mathrm{C}$ en jours longs de $16 \mathrm{~h}$ au niveau du bourgeon apical de la zone de l'axe qui lui est sousjacente et des limbes assimilateurs (fig 6). 


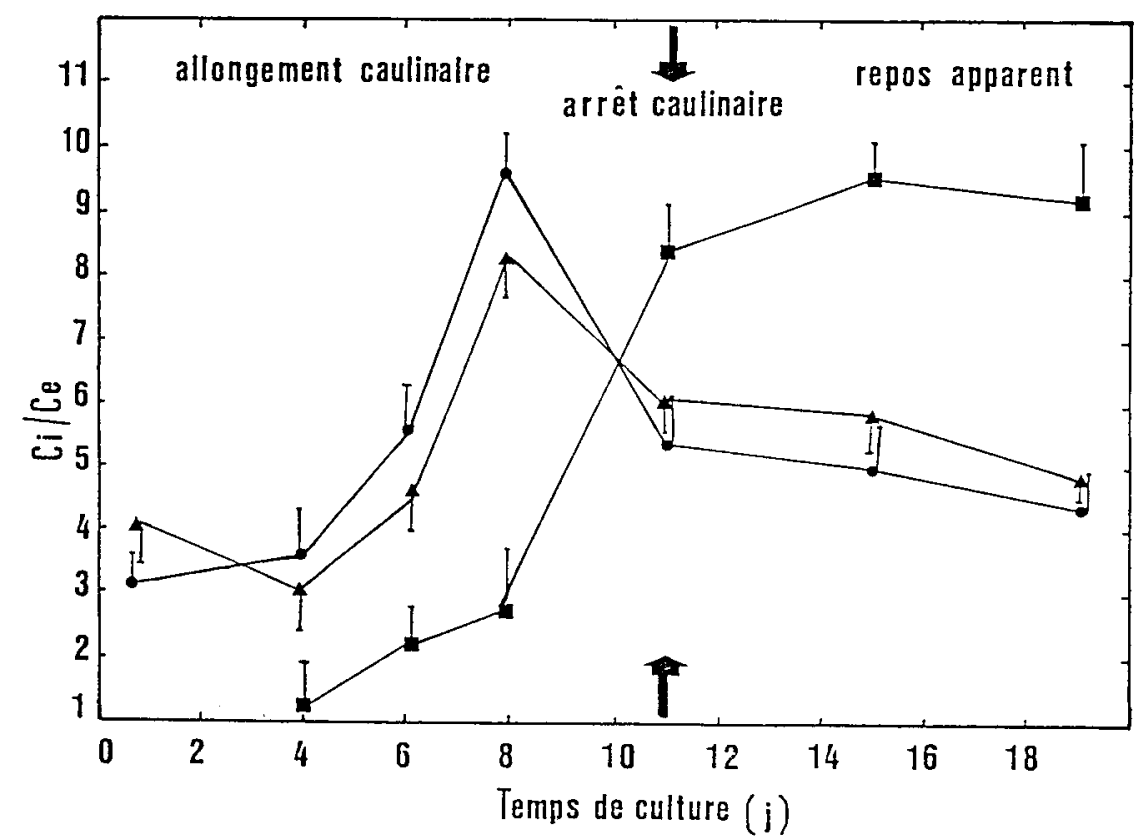

Fig 5. Evolution du rapport $\mathrm{Ci} / \mathrm{Ce}$ de la concentration intracellulaire à la concentration extracellulaire en DMO en fonction du temps en jours au cours de la $3^{8}$ vague de croissance chez le chêne pédonculé cultivé à $25^{\circ} \mathrm{C}$ en jours longs de $16 \mathrm{~h}$. Chaque valeur représente la moyenne de 12 mesures \pm erreur standard. N'en sont figurées que les moitiés. $\bullet$, bourgeon apical; $\mathbf{\Delta}$, axe sous-jacent au bourgeon apical; $\boldsymbol{\square}$, limbes assimilateurs.

\section{Le bourgeon apical}

Bien que l'apex encore entouré d'écailles, ne mesure que 2 à $3 \mathrm{~mm}$ et qu'il renferme un nombre élevé d'ébauches foliaires en début de croissance, la concentration en assimilats y est élevée $\left(45 \times 10^{3} \mathrm{dpm} \cdot \mathrm{mg}^{-1}\right.$ $\left.M^{\star}\right)$. Elle diminue faiblement ensuite jusqu'au $8^{\mathrm{e}}$ jour du flush. Au-delà du $8^{\mathrm{e}}$ jour, la radioactivité y diminue d'environ $50 \%$ pour devenir stable durant la phase de repos apparent. Donc la plus grande capacité d'accumulation du bourgeon apical correspond à la phase pendant laquelle le plastochrone est élevé et l'accumulation de la DMO forte.

* MF: matière fraîche

\section{L'axe sous-jacent}

Au début d'une vague de croissance, les valeurs observées dans l'axe sous-jacent au bourgeon terminal $\left(20 \times 10^{3} \mathrm{dpm} \cdot \mathrm{mg}^{-1}\right.$ MF) sont inférieures à celles de ce bourgeon. Elles s'élèvent fortement par la suite, jusqu'au $8^{e}$ jour du flush, où elles deviennent légèrement supérieures à celles du bourgeon terminal; la concentration en assimilats est alors doublée. Une baisse de $5 \%$ est ensuite observée au $14-18^{\circ}$ jour de la vague de croissance, le bourgeon terminal concentre de nouveau plus les assimilats que la zone de l'axe sous-jacente.

L'évolution notée avec le ${ }^{14} \mathrm{CO}_{2}$ est une nouvelle fois parallèle aux capacités d'ac- 


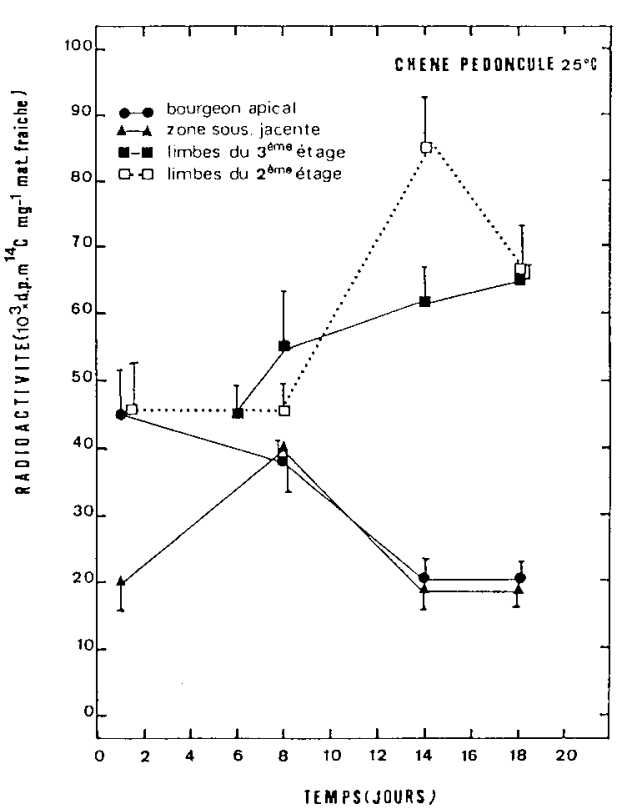

Fig 6. Distribution des assimilats (exprimée en $\mathrm{dpm}$ ) chez de jeunes plants de chêne pédonculé cultivés à $25^{\circ} \mathrm{C}$ en jours longs de $16 \mathrm{~h}$, au cours de la $3^{\theta}$ vague de croissance après une incorporation de ${ }^{14} \mathrm{CO}_{2}$ pendant $6 \mathrm{~h}$. Chaque valeur est la moyenne de 14 mesures associée à un intervalle de confiance pour un seuil de sécurité de $95 \%$. N'en sont figurées que les moitiés.

cumulation de la DMO observée précédemment.

\section{Les limbes assimilateurs de l'étage $n$ en formation}

II s'agit des limbes assimilateurs qui se forment et s'allongent au cours de la $3^{\theta}$ vague de croissance. Prélevés au $6^{\ominus}$ jour du flush, de longueur réduite (moins de 10 $\mathrm{mm}$ ), ils accumulent autant d'assimilats que le bourgeon apical. Leur capacité d'accumulation s'élève par la suite avec leur croissance et demeure supérieure à celle des autres territoires.

\section{Les limbes assimlateurs adultes de l'étage $n-1$}

Ils présentent des valeurs comparables à celles du bourgeon apical, d'environ $45 x$ $10^{3} \mathrm{dpm} \cdot \mathrm{mg}^{-1} \mathrm{MF}$, du $1^{\mathrm{er}}$ au $8^{\mathrm{e}}$ jour de la $3^{e}$ vague de croissance. Au-delà du $8^{\theta}$ jour, la concentration en photosynthétats s'élève, elle est multipliée par 2 au $14^{e}$ jour. Elle diminue en fin de vague et les feuilles du $2^{e}$ étage atteignent le même seuil d'assimilation photosynthétique que les limbes assimilateurs du $3^{e}$ étage, qui terminent leur étalement $\left(18^{\mathbf{e}}\right.$ jour de la vague de croissance).

\section{DISCUSSION ET CONCLUSIONS}

L'étude des relations entre la morphogenèse d'une plante et la croissance des feuilles est un thème classique de la physiologie du développement, par ailleurs toujours exploré (Miesh, 1990). Les travaux de Dostal ont ouvert la voie dans ce domaine à de nombreuses recherches (Dostal, 1967; Sebanek, 1985). L'étude des conséquences de l'ablation des feuilles ont abouti, chez les ligneux, à des résultats très importants. Ainsi, l'ablation réalisée à un stade précoce a permis de montrer que les très jeunes feuilles, encore incluses dans le bourgeon, commandent l'intensité de l'organogenèse apicale (Gleditsia triacanthos, Neville, 1968). Depuis ces recherches, l'ablation des ébauches foliaires émergeant du bourgeon apical en fonctionnement a permis d'obtenir une croissance continue non seulement chez le chêne pédonculé, mais chez tous les arbres dont la croissance rythmique a été étudiée (cacaoyer, Vogel, 1975; pommier, Zanette, 1981; manguier, Parisot, 1983; châtaignier, Si-Mohamed, 1983; Terminalia, Maillard, 1987). 
Les jeunes feuilles en croissance représentent un élément fondamental de la croissance rythmique du chêne pédonculé.

Ce résultat confirme les conclusions concernant la croissance rythmique de cette espèce faites par Champagnat et al (1986). Il est même permis de penser qu'il y a une valeur générale. L'utilisation de la ${ }^{14} \mathrm{C}$-DMO apporte des précisions nouvelles : par rapport à son incorporation intracellulaire, le bourgeon terminal est toujours l'organe dominant chez les plants dont on a supprimé toutes les jeunes feuilles. On peut donc envisager qu'il constitue le territoire qui draine à lui les nutriments. En fait, il ne subit qu'une concurrence affaiblie de la part des tissus de l'axe, sous-jacents, contrairement à ce qui a lieu chez les plantes intactes. Si on doit accorder un crédit à la valeur du pH intracellulaire déterminé par la méthode de la ${ }^{14}$ C-DMO (Candelier, 1989; Beffagna et Romani, 1989), le pH intracellulaire moyen des cellules composant le bourgeon terminal reste supérieur à celui des cellules composant les tissus caulinaires, porteurs de ce bourgeon chez les plantes opérées. Situation qui s'avère favorable à une organogenèse constante de la part du bourgeon terminal. Ce résultat est un argument supplémentaire à notre hypothèse qui privilégie, vis-à-vis de la croissance rythmique du chêne, des corrélations à très courte distance entre les différents territoires composant l'extrémité de la pousse en croissance : apex, tissus de l'axe sousjacents au bourgeon terminal, jeunes feuilles (Barnola et al, 1986). Chacun de ces territoires est pour nous un puits. L'expansion de l'un n'est finalement possible que si l'expansion des deux autres devient plus faible. Dans le cas de plants régulièrement et très tôt effeuillés, le puits «jeunes feuilles» est supprimé et le puits “méristème terminal» devient prépondérant. La croissance peut alors être continue. Notre hypothèse est proche de cette réflexion de El Morsy et Millet (1989) qui écrivent à propos de la croissance rythmique de Citrus deliciosa que le "déterminisme de la croissance périodique doit être recherché dans le mode de fonctionnement du méristème terminal".

Les feuilles ayant atteint leur taille déffnitive participent à l'élongation du jeune plant. La croissance en longueur de l'étage $n+1$ est, pour une grande part, dépendante de l'intégrité follaire de l'étage $n$.

La forte diminution de l'allongement est à mettre au compte du déficit en composés, vraisemblablement glucidiques et lipidiques, nécessaires au grandissement cellulaire et non pas à une diminution du nombre de cellules formées au niveau d'un entre-nceud. En effet, lorsque l'étage $n$ est effeuillé, les entre-nœuds qui constituent l'étage $n+1$ sont déjà initiés avec toutes leurs caractéristiques cellulaires (Champagnat et al, 1986).

L'incorporation intracellulaire de la ${ }^{14} \mathrm{C}$ DMO marque uniquement les potentialités de croissance des entre-nœuds et non pas leur croissance effective. Les potentialités subsistent même si les éléments nécessaires à cette croissance font ensuite défaut. Dès lors, le marquage par la DMO n'est pas modifié et reste comparable à celui effectué avec des plants non effeuillés.

Ces résultats montrent en outre que l'élongation des entre-nœuds est beaucoup plus aisée à modifier que l'organogenèse ou même le grandissement foliaire.

En fait, les feuilles d'un étage contribuent surtout à l'élongation des premiers entre-nœuds de l'étage suivant, qui sont 
associés à des stipules et à la première feuille à limbe assimilateur. Ce sont pour ces territoires que les pourcentages de réduction de croissance obtenus sont les plus forts.

Mais la croissance de l'entre-nœud de l'étage en formation dépend aussi de la feuille sus-jacente à cet entre-nœud.

Laisser une feuille de l'étage $n$, dont la surface double, permet d'obtenir un allongement de l'entre-nœud sous jacent. Les relations feuilles-croissance internodale sont reconnues depuis longtemps chez de nombreuses plantes herbacées et ligneuses (Millet, 1970; Crabbé, 1970; Millet et al, 1982; Maillard, 1987; Zobel, 1989). Notre résultat n'est donc pas nouveau, mais il devait être signalé dans une réflexion sur les facteurs intervenant dans l'élongation. En fait, il reste encore à comprendre pourquoi les derniers entre-nœuds formés restent très courts alors qu'ils sont associés à des feuilles à limbe entier. Des études sont en cours pour résoudre ce problème.

\section{L'existence de puits dont I'importance varie au cours d'un flush est confirmée par le marquage au ${ }^{14} \mathrm{CO}_{2}$}

Le marquage, au cours de la $3^{\theta}$ vague de croissance, donne des résultats dont l'évolution se calque sur celle obtenue avec la DMO. II montre que :

- le bourgeon apical est un centre d'appel fort au début de la vague de croissance;

- les tissus de l'axe sous-jacent au bourgeon apical sont à leur tour un centre d'appel important mais plus tardivement, au $\mathbf{8}^{\mathrm{e}}$ jour du flush;

- l'accumulation d'assimilats dans les jeunes limbes peut être repérée dès le $6^{\ominus}$ jour du flush. A ce moment, ils mesurent moins de $10 \mathrm{~mm}$ et la part de leur photosynthèse propre est certainement faible.
Ceci conforte l'hypothèse qu'elles constituent un puits important durant la $1^{\text {re }}$ semaine de la vague de croissance, à une période où l'hétéroblastie se met en place (Alatou et al, 1989). Enfin, notons que lorsque le bourgeon gonfle, au tout début de la vague de croissance, il renferme un nombre élevé de primordia en différenciation. Cette particularité est vraisemblablement responsable de la force d'appel du bourgeon noté précédemment.

Dickson (1989) a aussi employé cette même technique de marquage chez le chêne rouge, mais dans un autre but que l'étude des mécanismes à la base de la croissance rythmique de cet arbre. II a montré que les assimilats synthétisés par les feuilles de la $2^{\mathbf{e}}$ vague de croissance, sont utilisés à l'édification de la $3^{\theta}$. Son résultat rejoint donc celui que nous avons obtenu par ablation des feuilles de ce $2^{\theta}$ étage et que nous avons discuté précédemment.

En conclusion, la combinaison de techniques biologiques classiques couplées à des marquages biochimiques, nous amène à formuler une hypothèse claire des mécanismes de la croissance rythmique. Nous convenons que cette hypothèse s'éloigne notablement de l'hypothèse classique basée sur des concurrences en eau entre organes en croissance (Hallé et Martin, 1968). Mais peut-être que cet éloignement n'est qu'apparent, car les variations de teneur en eau sont en fait précédées par des fluctuations biochimiques cellulaires traduites par les variations d'incorporation de la DMO et de marquage au ${ }^{14} \mathrm{CO}_{2}$.

De plus, si nous avons invoqué des mécanismes faisant intervenir des puits bien localisés, nous n'avons pas évoqué les sources. Actuellement, des recherches sont en cours à ce sujet (Parmentier et al, 1990) qui montrent que les racines seraient l'une d'entre elles et participeraient par l'intermédiaire de régulateurs de crois- 
sance de type cytokinines, à une composante fondamentale du rythme de croissance : l'hétéroblastie.

\section{RÉFÉRENCES}

Alatou D, Barnola P, Lavarenne S, Gendraud M (1989) Caractérisation de la croissance rythmique du chêne pédonculé. Plant Physiol Biochem 27, 275-280

Auriac MC (1987) Contribution à l'étude de la pénétration des sucres dans les cellules accumulatrices chez le Crosne du Japon (Stachys sieboldi Miq). Thèse Clermont-Ferrand II, $138 \mathrm{p}$

Auriac MC, Tort M (1985) Ultrastructural evidence for a direct transport from apoplast to vacuoles in the storage cells of Japanese artichoke. Physiol Veg 23, 301-307

Barnola P, Crochet A, Payan E, Gendraud M, Lavarenne $S$ (1986) Modification du métabolisme énergétique et de la perméabilité dans le bourgeon apical et l'axe sous-jacent au cours de l'arrêt de croissance momentané de jeunes plants de Chêne. Physiol Vég 24, 307-314

Beffagna N, Romani G (1989) Intracellular pH measurements in plant tissues. Suitability of the weak acid and weak base distribution method in Elodea densa leaves. Plant Physiol Biochem 27, 423-430

Candelier P, Dauphin G, Gendraud M (1989) In vivo ${ }^{31} \mathrm{P}$ nuclear magnetic resonance spectroscopy of different Helianthus tuberosus organs during the vegetative cycle. Plant Physiol Biochem 27, 1-7

Champagnat $P$, Payan $E$, Champagnat $M$, Barnola P, Lavarenne S, Bertholon C (1986) La croissance rythmique de jeunes chênes pédonculés cultivés en conditions contrôlées et uniformes. Colloque international sur l'arbre Naturalia monspeliensia, 303-337

Crabbé J (1970) Influences foliaires sur la croissance de la pousse annuelle du pommier. II. Effets de la suppression de jeunes feuilles sur la levée d'inhibition et le développement des bourgeons axillaires. Bull Rech Agron Gembloux 4, 206-219

Dickson RE (1989) Carbon and nitrogen allocation in trees. Forest Trees Physiology. Proc of the International Symposium 25-30 September 1988, Nancy, France. Ann Sci For 631s-647s.

Dickson RE, Nelson EA (1982) Fixation and distribution of ${ }^{14} \mathrm{C}$ in Populus deltoïdes during dormancy induction. Plant Physiol 54, 393401

Dickson RE, Shive JB (1982) ${ }^{14} \mathrm{CO}_{2}$ fixation, translocation and carbon metabolism in rapidly expanding leaves of Populus deltoïdes. Ann Bot (Lond) 50, 37-47

Dostal $R$ (1967) On integration in Plants. Harvard University Press, Cambridge, MA, 218 p

Gendraud M, Lafleuriel J (1983) Caractéristiques de l'absorption du saccharose et du tétraphénylphosphonium par les parenchymes de tubercules de topinambour, dormants et non dormants, cultivés in vitro. Physiol Vég 21, 1125-1133

Hallé F, Martin R (1968) Etude de la croissance rythmique de l'Hévéa (Hevea brasiliensis Müll Arg, Euphorbiacées crotonoidées). Adansonia 2, 475-503

Hansen P, Grauslund J (1973) ${ }^{14} \mathrm{C}$ studies on appletrees. VIII. The seasonal variation and nature of reserves. Plant Physiol 28, 24-32

Jefford TG, Edelman J (1961) Changes in content and composition of the fructose polymers in tubers of Helianthus tuberosus $L$ during growth of daughter plants. $J$ Exp Bot 12, 177-187

Kandiah S (1979) Turn over of carbohydrates in relation to growth in apple trees. II. Distribution of ${ }^{14} \mathrm{C}$ assimilates labeled in autumn, spring and summer. Am J Bot 44, 185-195

Kurkdjian A, Guern J (1978) Intracellular pH in higher plants cells. Improvement in the use of the 5-5' dimethyloxazolidine-2 $\left({ }^{14} \mathrm{C}\right)$, 4-dione distribution techniques. Plant Sci Lett 11, 337-344

Lacointe A (1989) Assimilate allocation and carbon reserves in Juglans regia L. seedlings. Proceedings of the International Symposium 25-30 septembre 1988, Nancy, France. Ann Sci For 832s-836s

Langenfeld-Heyser R (1987) Distribution of leaf assimilates in the stem of Picea abies L. Trees 1, 102-109

Lavarenne S (1965) Recherches sur la croissance des bourgeons du Chêne et de quelques autres espèces ligneuses. Ann Sci For $22,1-203$ 
Maillard P (1987) Etude du développement végétatif du Terminalia superba Englers et Diels en conditions contrôlées : mise en évidence de rythmes de croissance. Thèse, Paris VI, 205 p

Miesh R (1990) Morphogenèse de deux espèces à feuilles composées pennées, Guarea guidonia et Lycopersicon esculentum;potentialités exprimées et enseignements phylogénétiques. Thèse, Strasbourg, $165 \mathrm{p}$

Millet B (1970) Analyse des rythmes de croissance de la Fève (Vicia faba L.). Thèse, Besançon, $132 \mathrm{p}$

Mokhbi A (1988) Etude des régulateurs de croissance et dormance chez le Frêne. Thèse, Nice

Neville P (1968) Morphogenèse chez Gleditsia triacanthos L. I. Mise en évidence expérimentale de corrélations jouant un rôle dans la morphogenèse et la croissance des bourgeons et des tiges. Ann Sci Nat Bot Série 9, 433-510

Parisot E (1983) Etude de la croissance rythmique chez de jeunes manguiers (Manguifera indica L.). Thèse, Clermont-Ferrand II

Parmentier C, Barnola P, Maillard P, Lavarenne $S$ (1990) Etude de la croissance rythmique du Chêne pédonculé, influence du système racinaire. Colloque international sur l'arbre, Montpellier, France
Payan E (1982) Contribution à l'étude de la croissance rythmique chez des chênes pédonculés (Quercus pedunculata Ehrh). Thèse, Clermont-Ferrand II

Pezet-Si-Mohamed $Y$ (1987) Caractérisation des potentialités morphogènes du Châtaignier (Castanea sativa Miller). Distribution et possibilités de translocation des réserves insolubles et solubles associées à des gradients de $\mathrm{pH}$ intracellulaires. Thèse, Clermont-Ferrand II

Sebanek J (1985) Scientific heritage of Pr Dostal for the development of experimental plant morphology. Acta Univ Agric Fac Agron 33, 25-69

Si-Mohamed C (1983) Germination, rythmes de croissance et morphogenèse de jeunes plants chez Castanea sativa Miller. Thèse Clermont-Ferrand II, $201 p$

Vogel M (1975) Recherches du déterminisme du rythme de croissance du Cacaoyer. Café Cacao The 19, 265-290

Zanette F (1981) Recherches descriptives et expérimentales sur la morphogenèse des systèmes aériens et racinaires de quelques porte-greffes du Pommier. Thèse, ClermontFerrand II, $159 \mathrm{p}$

Zobel AM (1989) Origin of nodes and internodes in plants shoots. I. Transverse zonation of apical parts of the shoot. Ann Sci Bot 63, 201-208 